\title{
Multi-marginal maximal monotonicity and convex analysis
}

\author{
Sedi Bartz; Heinz H. Bauschke”, Hung M. Phan; and Xianfu Wang ${ }^{\S}$
}

September 12, 2019

\begin{abstract}
Monotonicity and convex analysis arise naturally in the framework of multi-marginal optimal transport theory. However, a comprehensive multi-marginal monotonicity and convex analysis theory is still missing. To this end we study extensions of classical monotone operator theory and convex analysis into the multi-marginal setting. We characterize multi-marginal $c$-monotonicity in terms of classical monotonicity and firmly nonexpansive mappings. We provide Minty type, continuity and conjugacy criteria for multi-marginal maximal monotonicity. We extend the partition of the identity into a sum of firmly nonexpansive mappings and Moreau's decomposition of the quadratic function into envelopes and proximal mappings into the multi-marginal settings. We illustrate our discussion with examples and provide applications for the determination of multi-marginal maximal monotonicity and multi-marginal conjugacy. We also point out several open questions.
\end{abstract}

2010 Mathematics Subject Classification: Primary 47H05, 26B25; Secondary 49N15, 49K30, 52A01, 91B68.

Keywords: $c$-convexity, $c$-monotonicity, $c$-splitting set, cyclic monotonicity, Kantorovich duality, maximal monotonicity, Minty Theorem, Moreau envelope, multi-marginal, optimal transport.

\section{Introduction}

Our discussion stems from multi-marginal optimal transport theory: Let $\left(X_{1}, \mu_{1}\right), \ldots,\left(X_{N}, \mu_{N}\right)$ be Borel probability spaces. We set $X=X_{1} \times \cdots \times X_{N}$ and we denote by $\Pi(X)$ the set of all Borel probability measures $\pi$ on $X$ such that the marginals of $\pi$ are the $\mu_{i}$ 's. Let $c: X \rightarrow \mathbb{R}$

*Mathematics, University of Massachusetts Lowell, MA 01854, USA. E-mail: sedi_bartz@uml .edu.

${ }^{\dagger}$ Mathematics, University of British Columbia, Kelowna, B.C. V1V 1V7, Canada. E-mail: heinz . bauschke@ubc.ca.

${ }_{\ddagger}^{\ddagger}$ Mathematics, University of Massachusetts Lowell, MA 01854, USA. E-mail: hung_phan@uml .edu.

$\S$ Mathematics, University of British Columbia, Kelowna, B.C. V1V 1V7, Canada. E-mail: shawn. wang@ubc.ca. 
be a cost function. A cornerstone of multi-marginal optimal transport theory is Kellerer's [16] generalization of the Kantorovich duality theorem to the multi-marginal case. Kellerer's duality theorem asserts that, in a suitable framework,

$$
\min _{\pi \in \Pi(X)} \int_{X} c(x) d \pi(x)=\max _{\substack{u_{i} \in L_{1}\left(\mu_{i}\right), \sum_{1 \leq i \leq N} u_{i} \leq c}} \sum_{1 \leq i \leq N} \int_{X_{i}} u_{i}\left(x_{i}\right) d \mu_{i}\left(x_{i}\right) .
$$

It follows that if $\pi$ is a solution of the left-hand side of $(1)$ and $\left(u_{1}, \ldots, u_{N}\right)$ is a solution of the right-hand side of (1), then $\pi$ is concentrated on the subset $\Gamma$ of $X$ where the equality $c=\sum_{1 \leq i \leq N} u_{i}$ holds. In recent publications (see, for example, $[5,15,17])$ such subsets $\Gamma$ of $X$ are referred to as $c$-splitting sets: Let $N \geq 2$ be a natural number and $I=\{1, \ldots, N\}$ an index set. Let $X_{1}, \ldots, X_{N}$ be nonempty sets, $X=X_{1} \times \cdots \times X_{N}$ and $c: X \rightarrow \mathbb{R}$ a function.

Definition 1.1 (c-splitting set) Let $\Gamma \subseteq X$. We say that $\Gamma$ is a $c$-splitting set if for each $i \in I$ there exists a function $\left.\left.u_{i}: X_{i} \rightarrow\right]-\infty,+\infty\right]$ such that

$$
\forall x=\left(x_{1}, \ldots, x_{N}\right) \in X, \quad c\left(x_{1}, \ldots, x_{N}\right) \leq\left(\bigoplus_{i \in I} u_{i}\right)(x):=\sum_{i \in I} u_{i}\left(x_{i}\right)
$$

and

$$
\forall x=\left(x_{1}, \ldots, x_{N}\right) \in \Gamma, \quad c\left(x_{1}, \ldots, x_{N}\right)=\left(\bigoplus_{i \in I} u_{i}\right)(x):=\sum_{i \in I} u_{i}\left(x_{i}\right) .
$$

In this case we say that $\left(u_{1}, \ldots, u_{N}\right)$ is a c-splitting tuple of $\Gamma$. Given functions $\left.\left.u_{i}: X_{i} \rightarrow\right]-\infty,+\infty\right]$ that satisfy (2), we call the set of all points $\left(x_{1}, \ldots, x_{N}\right) \in X$ that satisfy (3) the c-splitting set generated by the tuple $\left(u_{1}, \ldots, u_{N}\right)$.

In the case $N=2$, splitting sets are natural in convex analysis as graphs of subdifferentials. Indeed, by the Young-Fenchel inequality the graph of the subdifferential $\partial f$ is the $c$-splitting set generated by the pair $\left(f, f^{*}\right)$ where $c=\langle\cdot, \cdot\rangle$ is the classical pairing between a linear space and its dual. Similar to the two-marginal case, in the multi-marginal case monotonicity arises naturally as well:

Definition 1.2 (c-cyclic monotonicity) The subset $\Gamma$ of $X$ is said to be c-cyclically monotone of order $n$, $n$-c-monotone for short, if for all $n$ tuples $\left(x_{1}^{1}, \ldots, x_{N}^{1}\right), \ldots,\left(x_{1}^{n}, \ldots, x_{N}^{n}\right)$ in $\Gamma$ and every $N$ permutations $\sigma_{1}, \ldots, \sigma_{N}$ in $S_{n}$

$$
\sum_{j=1}^{n} c\left(x_{1}^{\sigma_{1}(j)}, \ldots, x_{N}^{\sigma_{N}(j)}\right) \leq \sum_{j=1}^{n} c\left(x_{1}^{j}, \ldots, x_{N}^{j}\right)
$$

$\Gamma$ is said to be c-cyclically monotone if it is $n$-c-monotone for every $n \in\{2,3, \ldots\}$; and $\Gamma$ is said to be

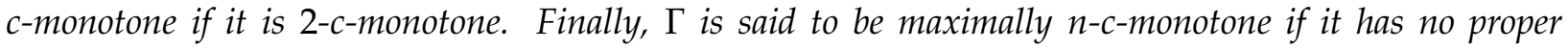
n-c-monotone extension. 
Cyclic monotonicity was first introduced by Rockafellar [24] in the framework of classical convex analysis. During the late 80 s and early 90 s (see $[8,23,26])$ the concept was generalized to $c$-cyclic monotonicity in order to hold for more general cost functions $c$ in the framework of twomarginal optimal transport theory. Currently, it lays at the foundations of the theory (see for example [11, 28, 30]) and plays a role also in recent refinements (see, for example, [2, 3]). Extending the role it plays in two-marginal optimal transport theory, in the past two and a half decades multi-marginal $c$-monotonicity and aspects of $c$-convex analysis are becoming an integral part of the fast evolving multi-marginal optimal transport theory as can be seen, for example, in $[1,5,7,9,10,12,13,14,15,17,18,19,20,21,22,27]$. An important instance of an extension from the two-marginal case relating Definition 1.1 with Definition 1.2 is the known fact that $c$-splitting sets are $c$-cyclically monotone (see, for example, $[5,15,17,18])$.

Before attending our convex analytic discussion we remark that in order to make optimal transport compatible with our discussion, one should exchange min for max in the left-hand side of (1), exchange max for min in the right-hand side of (1) and, finally, exchange the constraint $\sum_{i} u_{i} \leq c$ in the right-hand side of (1) with the constraint $c \leq \sum_{i} u_{i}$ as we did in Definition 1.1 and Definition 1.2.

In the framework of multi-marginal optimal transport, presumably the most traditional and well studied cost functions are classical extensions of the pairing between a linear space and its dual:

For the remainder of our discussion, for each $1 \leq i \leq N$, we assume that $X_{i}=H$ is a real Hilbert space with inner product $\langle\cdot, \cdot\rangle$ and induced norm $\|\cdot\|$. We let $c: X \rightarrow \mathbb{R}$ be the cost function defined by

$$
c\left(x_{1}, \ldots, x_{N}\right)=\sum_{1 \leq i<j \leq N}\left\langle x_{i}, x_{j}\right\rangle .
$$

It follows from straightforward computation (see for example [5]) that a set $\Gamma \subseteq X$ is $n$-c-monotone if and only if it is $n-c$-monotone with respect to each of the functions

$$
\left(x_{1}, \ldots, x_{N}\right) \mapsto-\sum_{1 \leq i<j \leq N} \frac{1}{2}\left\|x_{i}-x_{j}\right\|^{2} \quad \text { and } \quad\left(x_{1}, \ldots, x_{N}\right) \mapsto \frac{1}{2}\left\|\sum_{i=1}^{N} x_{i}\right\|^{2} .
$$

Although classical convex analysis and monotonicity are instrumental in multi-marginal optimal transport, and although several multi-marginal convex analytic results are already available (as we recall in our more specific discussion further below), to the best of our knowledge, a comprehensive multi-marginal monotonicity and convex analysis theory is still lacking. To this end, in the present paper we lay additional foundations and provide several extensions of classical monotone operator theory and convex analysis into the multi-marginal settings.

The remainder of the paper is organized as follows. In Section 2 we provide a characterization of multi-marginal $c$-monotonicity in terms of classical monotonicity. We employ this characterization in order to provide several equivalent criteria, including a Minty-type criterion, a criterion based 
on the partition of the identity into a sum of firmly nonexpansive mappings, and other criteria for multi-marginal maximal c-monotonicity. In Section 3 we provide a continuity criterion for multimarginal maximal monotonicity. In Section 4 we focus on multi-marginal convex analysis. In particular, we extend Moreau's decompositions and provide criteria for maximal c-monotonicity of $c$-splitting sets, the multi-marginal extensions of subdifferentials. We show that the same criteria also imply multi-marginal $c$-conjugacy of $c$-splitting functions. In the case $N=3$ we also provide a class of $c$-splitting triples for which $c$-conjugacy implies maximal $c$-monotonicity. Section 5 contains examples and applications of our results to the problem of determining maximal $c$-monotonicity of sets and c-conjugacy of $c$-splitting tuples, thus reducing the need of further challenging computations of multi-marginal $c$-conjugate tuples. Additionally, we point out several open problems.

In the remainder of this section we collect standard notations and preliminary facts from classical monotone operator theory and convex analysis which, largely, follow [6]. Let $A: H \rightrightarrows H$ be a set-valued mapping. The domain of $A$ is the set $\operatorname{dom} A=\{x \in H \mid A x \neq \varnothing\}$. The range of $A$ is the set $\operatorname{ran} A=A(H)=\bigcup_{x \in H} A x$, the graph of $A$ is the set gra $A=\{(x, u) \in H \times H \mid u \in A x\}$ and the inverse mapping of $A$ is the mapping $A^{-1}$ satisfying $x \in A^{-1} u \Leftrightarrow u \in A x$. $A$ is said to be monotone if

$$
(\forall(x, u) \in \operatorname{gra} A)(\forall(y, v) \in \operatorname{gra} A) \quad\langle x-y, u-v\rangle \geq 0 .
$$

$A$ is said to be maximally monotone if there exists no monotone operator $B$ such that gra $A$ is a proper subset of gra $B$. The resolvent of $A$ is the mapping $J_{A}=(A+\mathrm{Id})^{-1}$ where Id is the identity mapping. The mapping $T: \operatorname{dom} T \subseteq H \rightarrow H$ is said to be firmly nonexpansive if

$$
(\forall x \in \operatorname{dom} T)(\forall y \in \operatorname{dom} T) \quad\|T x-T y\|^{2}+\|(\operatorname{Id}-T) x-(\operatorname{Id}-T) y\|^{2} \leq\|x-y\|^{2},
$$

where $\operatorname{dom} T \subseteq H$. The function $f: H \rightarrow]-\infty,+\infty]$ is said to be proper if $\operatorname{dom} f:=\{x \in$ $H \mid f(x)<\infty\} \neq \varnothing$. The Fenchel conjugate of the function $f$ is the function $f^{*}$ defined by

$$
f^{*}(u)=\sup _{x \in H}(\langle u, x\rangle-f(x))
$$

We set $q(\cdot)=\frac{1}{2}\|\cdot\|^{2}$. The Moreau envelope of $f$ is the function defined by the infimal convolution

$$
e_{f}(s)=(f \square q)(s)=\inf _{x \in H}(f(x)+q(s-x)) .
$$

The subdifferential of the proper function $f$ is the mapping $\partial f: H \rightrightarrows H$ defined by

$$
\partial f(x)=\{u \in H \mid f(x)+\langle u, y-x\rangle \leq f(y), \forall y \in H\} .
$$

The indicator function of a subset $C$ of $H$ is the function $\left.\left.\iota_{C}: H \rightarrow\right]-\infty,+\infty\right]$ which vanishes on $C$ and equals $+\infty$ on $H \backslash C$.

Fact 1.3 (Minty's Theorem [6, Theorem 21.1]) Let $A: H \rightrightarrows H$ be monotone. Then $A$ is maximally monotone if and only if $\operatorname{ran}(\mathrm{Id}+A)=H$.

Fact 1.4 ([6, Proposition 23.8]) Let $A: H \rightrightarrows H$. Then 
(i) $J_{A}$ is firmly nonexpansive if and only if $A$ is monotone;

(ii) $J_{A}$ is firmly nonexpansive and $\operatorname{dom} J_{A}=H$ if and only if $A$ is maximally monotone.

Let $f$ be a proper lower semicontinuous convex function. The proximity operator [6, Definition 12.23] of $f$ is defined by

$$
\operatorname{Prox}_{f}: H \rightarrow H: x \mapsto \operatorname{Prox}_{f} x=\underset{y \in H}{\operatorname{argmin}}(f(y)+q(y-x)) .
$$

For all $s \in H,[6$, Proposition 12.15] implies that there is a unique minimizer of $f(\cdot)+q(s-\cdot)$ over all $x \in H$; thus, the proximity operator of $f$ is well defined. Furthermore, we also have $\operatorname{Prox}_{f}=J_{\partial f}$.

Additional properties of the Moreau envelope are:

Fact 1.5 (Moreau envelope) Let $f$ be a proper lower semicontinuous convex function. The following assertions hold:

(i) (Moreau decomposition) $e_{f}+e_{f^{*}}=q$.

(ii) $x=\operatorname{Prox}_{f} s \Leftrightarrow e_{f}(s)=f(x)+q(s-x)$.

(iii) $\left(\left[6\right.\right.$, Proposition 12.30]) $e_{f}$ is Fréchet differentiable with $\nabla e_{f}=\mathrm{Id}-\operatorname{Prox}_{f}$.

Finally, we set the marginal projections $P_{i}: X \rightarrow X_{i}:\left(x_{1}, \ldots, x_{N}\right) \mapsto x_{i}$ for $i$ in $\{1, \ldots, N\}$ and the two-marginal projections $P_{i, j}: X \rightarrow X_{i} \times X_{j}:\left(x_{1}, \ldots, x_{N}\right) \mapsto\left(x_{i}, x_{j}\right)$ for $i<j$ in $\{1, \ldots, N\}$. Given a subset $\Gamma$ of $X$, we set

$$
\Gamma_{i}=P_{i}(\Gamma) \quad \text { and } \quad \Gamma_{i, j}=P_{i, j}(\Gamma)
$$

We also define $A_{i, j}: X_{i} \rightrightarrows X_{j}$ via

$$
\operatorname{gra} A_{i, j}=\Gamma_{i, j}
$$

The notation $A_{i}$ is reserved for a different purpose and introduced in Section 2.

\section{A characterization of multi-marginal $c$-monotonicity and Minty type criteria for $c$-monotonicity}

Let $S: H \times H \rightarrow H$ be the mapping defined by $S(x, y)=x+y$. For any mapping $A: H \rightrightarrows H$, we have the identity [25, Lemma 12.14]

$$
J_{A^{-1}}=\mathrm{Id}-J_{A} \text {. }
$$

If, in addition, $A$ is monotone, then by Fact $1.4, J_{A}$ and $J_{A^{-1}}$ are single-valued, thus,

$$
J_{A}+J_{A^{-1}}=\left.\operatorname{Id}\right|_{S(\operatorname{gra} A)},
$$


which is equivalent to gra $A$ being parameterized by

$$
\operatorname{gra} A=\left\{\left(J_{A} s, J_{A^{-1}} s\right) \mid s \in S(\operatorname{gra} A)\right\} .
$$

Given a set $\Gamma \subseteq X$, we now associate with $\Gamma$ monotone mappings as follows.

Definition 2.1 Let $\Gamma \subseteq X$ be a set. For each index set $\varnothing \neq K \subsetneq I$, we define the mapping $A_{K}: H \rightrightarrows H$ by

$$
\operatorname{gra} A_{K}=\left\{\left(\sum_{i \in K} x_{i}, \sum_{i \in I \backslash K} x_{i}\right) \mid\left(x_{1}, \ldots, x_{N}\right) \in \Gamma\right\}
$$

and for each $i \in I$ we set $A_{i}=A_{\{i\}}$.

Our first aim is to characterize the $c$-monotonicity of a set $\Gamma$ in terms of the monotonicity of its $A_{K}$ 's, and furthermore, extend (10) and (11) to the multi-marginal settings. To this end we will employ the sum mapping

$$
S: X \rightarrow H:\left(x_{1}, \ldots, x_{N}\right) \mapsto \sum_{i \in I} x_{i}
$$

and the following fact which follows by a straightforward computation (see, e.g., [5, Fact 3.3]).

Fact 2.2 Let $x \in X$. If the subset $\Gamma$ of $X$ is $n$-c-cyclically monotone, then so is $\Gamma+x$.

Lemma 2.3 Let $\Gamma \subseteq X$ be a set. Then the following assertions are equivalent:

(i) $\Gamma$ is c-monotone;

(ii) For each $\varnothing \neq K \subsetneq I$, the mapping $A_{K}$ is monotone;

(iii) For each $\varnothing \neq K \subsetneq I$, the mapping $J_{A_{K}}: S(\Gamma) \rightarrow H$ is firmly nonexpansive.

In this case,

$$
J_{A_{1}}+\cdots+J_{A_{N}}=\left.\operatorname{Id}\right|_{S(\Gamma)}
$$

equivalently, $\Gamma$ can be parameterized by

$$
\Gamma=\left\{\left(J_{A_{1}} s, \ldots, J_{A_{N}} s\right) \mid s \in S(\Gamma)\right\}
$$

and, furthermore, for each $\varnothing \neq K \subsetneq I$,

$$
J_{A_{K}}=\sum_{i \in K} J_{A_{i}}
$$

Proof. (i) $\Leftrightarrow$ (ii): First we characterize the $c$-monotone relations of the set $\{z, 0\}$ in $X$. We employ a similar computation to the one in [5, Lemma 4.1]: For $z=\left(z_{1}, \ldots, z_{N}\right) \in X$ and $\varnothing \neq K \subsetneq I$ we set $z^{K}=\left(z_{1}^{K}, \ldots, z_{N}^{K}\right) \in X$ by

$$
z_{i}^{K}= \begin{cases}z_{i,} & i \in K \\ 0, & i \in I \backslash K\end{cases}
$$


From Definition 1.2 it follows that $\{z, 0\}$ is $c$-monotone if and only if for each $\varnothing \neq K \subsetneq I$

$$
\begin{aligned}
0 & \leq c(z)+c(0)-c\left(z^{K}\right)-c\left(z^{I \backslash K}\right) \\
& =\sum_{i, j \in I, i<j}\left\langle z_{i}, z_{j}\right\rangle+0-\sum_{i, j \in I, i<j}\left\langle z_{i}^{K}, z_{j}^{K}\right\rangle-\sum_{i, j \in I, i<j}\left\langle z_{i}^{I \backslash K}, z_{j}^{I \backslash K}\right\rangle \\
& =\sum_{i, j \in I, i<j}\left\langle z_{i}, z_{j}\right\rangle-\sum_{i, j \in K, i<j}\left\langle z_{i}, z_{j}\right\rangle-\sum_{i, j \in I \backslash K, i<j}\left\langle z_{i}, z_{j}\right\rangle=\left\langle\sum_{i \in K} z_{i}, \sum_{i \in I \backslash K} z_{i}\right\rangle .
\end{aligned}
$$

In general, from Definition 1.2 it follows that the set $\Gamma \subseteq X$ is $c$-monotone if and only if for any $x \in \Gamma$ and $y \in \Gamma$, the set $\{x, y\}$ is $c$-monotone, which, in turn, by invoking Fact 2.2, is equivalent to the set $\{x-y, 0\}$ being $c$-monotone. Summing up, we see that $\Gamma$ is $c$-monotone if and only if for any $x=\left(x_{1}, \ldots, x_{N}\right), y=\left(y_{1}, \ldots, y_{N}\right) \in \Gamma$ and any $\varnothing \neq K \subsetneq I$, by letting $z=x-y$,

$$
0 \leq\left\langle\sum_{i \in K} x_{i}-\sum_{i \in K} y_{i}, \sum_{i \in I \backslash K} x_{i}-\sum_{i \in I \backslash K} y_{i}\right\rangle,
$$

i.e., $A_{K}$ is monotone.

(ii) $\Leftrightarrow$ (iii): By the definition of $A_{K}$, it follows that dom $J_{A_{K}}=S(\Gamma)$. Thus, the equivalence (ii) $\Leftrightarrow$ (iii) follows immediately from Fact 1.4(i).

Finally, (14), (15) and (16) follow from (iii) and the definition of $A_{K}$.

We now address maximal $c$-monotonicity. Equivalent statements of Minty's characterization are: Let $A: H \rightrightarrows H$ be a monotone mapping. Then $A$ is maximally monotone if and only if

$$
S(\operatorname{gra}(A))=H,
$$

equivalently,

$$
\operatorname{gra}(A)+\operatorname{gra}(-\mathrm{Id})=H \times H .
$$

In order to extend our discussion of these formulas into the multi-marginal settings we will employ the following definitions and notations. We denote by $\Delta$ the subset of $X=X_{1} \times \cdots \times X_{N}$ defined by

$$
\Delta=\{(x, \ldots, x) \mid x \in H\} . \quad \text { Consequently, } \quad \Delta^{\perp}=\left\{\left(x_{1}, \ldots, x_{N}\right) \in X \mid \sum_{i=1}^{N} x_{i}=0\right\} .
$$

Corollary 2.4 Let $\Gamma \subseteq X$ be a c-monotone set. Then for every $u, v \in \Gamma$,

$$
u-v \in \Delta^{\perp} \quad \Leftrightarrow \quad u=v .
$$

Proof. Let $u=\left(u_{1}, \ldots, u_{N}\right)$ and $v=\left(v_{1}, \ldots, v_{N}\right)$ belong to $\Gamma$ and suppose that

$$
u-v=d=\left(d_{1}, \ldots, d_{N}\right) \in \Delta^{\perp} .
$$


We prove that $d_{i}=0$ for each $1 \leq i \leq N$. To this end, set $1 \leq i_{0} \leq N$. By Lemma 2.3, $A_{i_{0}}$ is monotone. Consequently we see that

$$
0 \leq\left\langle u_{i_{0}}-v_{i_{0}}, \sum_{i \neq i_{0}} u_{i}-\sum_{i \neq i_{0}} v_{i}\right\rangle=\left\langle d_{i_{0}}, \sum_{i \neq i_{0}} d_{i}\right\rangle=\left\langle d_{i_{0}},-d_{i_{0}}\right\rangle=-\left\|d_{i_{0}}\right\|^{2} \leq 0 .
$$

Combining Lemma 2.3 and Corollary 2.4 with classical two-marginal monotone operator theory, we arrive at the following result.

Theorem 2.5 (multi-marginal maximal c-monotonicity) Let $\Gamma \subseteq X$ be a c-monotone set. Then the following assertions are equivalent:

(i) For each $\varnothing \neq K \subsetneq I$ the mapping $A_{K}$ defined by (12) is maximally monotone;

(ii) There exists $\varnothing \neq K \subsetneq I$ such that the mapping $A_{K}$ is maximally monotone;

(iii) $\Gamma+\Delta^{\perp}=X$;

(iv) $J_{A_{1}}+\cdots+J_{A_{N}}=\mathrm{Id}$;

(v) For each $\varnothing \neq K \subsetneq I$ the firmly nonexpansive mapping $J_{A_{K}}: H \rightarrow H$ has full domain and $J_{A_{K}}=$ $\sum_{i \in K} J_{A_{i}} ;$

(vi) $S(\Gamma)=H$.

In this case, $\Gamma$ is maximally c-monotone.

Proof. (i) $\Rightarrow$ (ii) is trivial.

(ii) $\Rightarrow$ (iii): Suppose that $A_{K}$ is maximally monotone and let $a=\left(a_{1}, \ldots, a_{N}\right) \in X$. We will prove that there exist $x=\left(x_{1}, \ldots, x_{N}\right) \in \Gamma$ and $d=\left(d_{1}, \ldots, d_{N}\right) \in \Delta^{\perp}$ such that $x+d=a$. Indeed, the maximal monotonicity of $A_{K}$ implies that $\operatorname{ran}\left(A_{K}+\mathrm{Id}\right)=H$. Consequently, by the definition of $A_{K}$, there exists $x=\left(x_{1}, \ldots, x_{N}\right) \in \Gamma$ such that $\sum_{i=1}^{N} x_{i}=\sum_{i=1}^{N} a_{i}$. For each $1 \leq i \leq N$ we let $d_{i}=a_{i}-x_{i}$. Then $\sum_{i=1}^{N} d_{i}=0$, that is $d=\left(d_{1}, \ldots, d_{N}\right) \in \Delta^{\perp}$ and $x+d=a$.

(iii) $\Rightarrow$ (iv): Fix $1 \leq i_{0} \leq N$. We prove that $A_{i_{0}}+$ Id is onto. Indeed, let $s \in H$. We prove that there exists $x=\left(x_{1}, \ldots, x_{N}\right) \in \Gamma$ such that $\left(x_{i_{0}}, s\right) \in \operatorname{gra}\left(A_{i_{0}}+\mathrm{Id}\right)$. Indeed, let $h=\left(h_{1}, \ldots, h_{N}\right) \in X$ such that $\sum_{i=1}^{N} h_{i}=s$. Then (iii) implies the existence of $x=\left(x_{1}, \ldots, x_{N}\right) \in \Gamma$ and $d=\left(d_{1}, \ldots, d_{N}\right) \in \Delta^{\perp}$ such that $x+d=h$. Consequently, $\sum_{i=1}^{N} x_{i}=s$ which implies that $\left(x_{i_{0}}, s\right)=\left(x_{i_{0}}, \sum_{i=1}^{N} x_{i}\right) \in \operatorname{gra}\left(A_{i_{0}}+\mathrm{Id}\right)$. Thus, since $A_{i_{0}}$ is monotone, we conclude that its resolvent $J_{A_{i}}$ is firmly nonexpansive and has full domain. This is true for each $1 \leq i_{0} \leq N$ and since for any $s \in H$ there exists $x=\left(x_{1}, \ldots, x_{N}\right) \in \Gamma$ such that $\sum_{i=1}^{N} x_{i}=s$, we conclude that $\sum_{i=1}^{N} J_{A_{i}}(s)=\sum_{i=1}^{N} x_{i}=s$, that is, (iv) holds. 
(iv) $\Rightarrow(\mathrm{v})$ : Since $A_{K}$ is monotone for every $\varnothing \neq K \subsetneq I$, the resolvent $J_{A_{K}}$ is firmly nonexpansive and (iv) implies it has full domain. Furthermore, by employing our notations from the previous step, we see that for every $s \in H, \sum_{i \in K} J_{A_{i}}(s)=\sum_{i \in K} x_{i}=J_{A_{K}}(s)$, that is, we have arrived at (v).

(v) $\Rightarrow$ (i): Let $\varnothing \neq K \subsetneq I$. Since the resolvent $J_{A_{K}}$ is firmly nonexpansive and has full domain, $A_{K}$ is maximally monotone.

Summing up, we have established (i) $\Rightarrow$ (ii) $\Rightarrow$ (iii) $\Rightarrow$ (iv) $\Rightarrow$ (v) $\Rightarrow$ (i).

(iv) $\Rightarrow$ (vi): Since for each $1 \leq i \leq N, \operatorname{dom}\left(J_{A_{i}}\right)=S(\Gamma)$, then (iv) $\Rightarrow$ (vi).

(vi) $\Rightarrow$ (iii): Suppose that $S(\Gamma)=H$ and let $y \in X$. Then there exist $x \in \Gamma$ such that $S(y)=S(x)$. Consequently, $y-x \in \Delta^{\perp}$, which implies that $y=x+(y-x) \in \Gamma+\Delta^{\perp}$.

Finally, we prove that (iii) implies the maximal $c$-monotonicity of $\Gamma$. Indeed, suppose that $u$ is $c$ monotonically related to $\Gamma$. We then write $u=d+v$ where $d \in \Delta^{\perp}$ and $v \in \Gamma$. Since $u, v \in \Gamma \cup\{u\}$ which is $c$-monotone and $u-v \in \Delta^{\perp}$, Corollary 2.4 implies that $u=v \in \Gamma$.

Remark 2.6 To the best of our knowledge, the question whether the multi-marginal generalization of the other direction of Minty's characterization of maximal monotonicity holds, namely, whether the maximal $c$-monotonicity of the set $\Gamma$ implies that $\Gamma+\Delta^{\perp}=X$, equivalently, that $J_{A_{1}}+\cdots+$ $J_{A_{N}}=\mathrm{Id}$, is still open.

Remark 2.7 In the partition of the identity in (14) and in Theorem 2.5(iv) we conclude from (16) and Theorem $2.5(\mathrm{v})$ that any partial sum of the firmly nonexpansive mappings is also firmly nonexpansive. This is not the case for general partitions of the identity into sums of firmly nonexpansive mappings; indeed, an example where partial sums of a partition of the identity into firmly nonexpansive mappings fail to be firmly nonexpansive is provided in [4, Example 4.4]. We elaborate further on this in Example 5.7 below.

\section{Multi-marginal maximal $c$-monotonicity via continuity}

In the classical two-marginal case an important class of maximally monotone operators is the one of continuous monotone operators. A continuity criterion guarantees maximality in the multimarginal framework as well:

Theorem 3.1 Let $\Gamma \subseteq X$ be a c-monotone set. Suppose that $\Gamma$ is the graph of a continuous mapping $T=\left(T_{2}, \ldots, T_{N}\right): X_{1} \rightarrow \Pi_{i=2}^{N} X_{i}$, i.e.,

$$
\Gamma=\operatorname{gra}(T)=\left\{\left(x, T_{2} x, \ldots, T_{N} x\right) \mid x \in H\right\}
$$

where for each $2 \leq i \leq N$ the mapping $T_{i}: H \rightarrow H$ is continuous. Then $\Gamma$ is maximally c-monotone.

We provide two proofs for Theorem 3.1. We begin with a direct proof. 
Proof. Let $u=\left(u_{1}, \ldots, u_{N}\right)$ be $c$-monotonically related to $\Gamma$. We prove that $u \in \Gamma$. Since $A_{1}$, induced from the $c$-monotone set $\Gamma \cup\{u\}$, is monotone,

$$
\forall x \in H, \quad 0 \leq\left\langle u_{1}-x, \sum_{i=2}^{N}\left(u_{i}-T_{i} x\right)\right\rangle .
$$

For $t>0$ we let $x_{t}=u_{1}+t \sum_{i=2}^{N}\left(u_{i}-T_{i} u_{1}\right)$. Then $x_{t} \longrightarrow u_{1}$ as $t \rightarrow 0^{+}$and

$$
0 \leq t\left\langle\sum_{i=2}^{N}\left(T_{i} u_{1}-u_{i}\right), \sum_{i=2}^{N}\left(u_{i}-T_{i} x_{t}\right)\right\rangle .
$$

Since each $T_{i}$ is continuous, we deduce that

$$
\begin{aligned}
0 & \leq\left\langle\sum_{i=2}^{N}\left(T_{i} u_{1}-u_{i}\right), \sum_{i=2}^{N}\left(u_{i}-T_{i} x_{t}\right)\right\rangle \\
& \stackrel{t \rightarrow 0^{+}}{\longrightarrow}\left\langle\sum_{i=2}^{N}\left(T_{i} u_{1}-u_{i}\right), \sum_{i=2}^{N}\left(u_{i}-T_{i} u_{1}\right)\right\rangle=-\left\|\sum_{i=2}^{N}\left(u_{i}-T_{i} u_{1}\right)\right\|^{2},
\end{aligned}
$$

which implies

$$
\sum_{i=2}^{N} u_{i}=\sum_{i=2}^{N} T_{i} u_{1}
$$

equivalently,

$$
\left(u_{1}, \ldots, u_{N}\right)-\left(u_{1}, T_{2} u_{1}, \ldots, T_{N} u_{1}\right) \in \Delta^{\perp} .
$$

Thus, by Corollary 2.4, we have $\left(u_{1}, \ldots, u_{N}\right)=\left(u_{1}, T_{2} u_{1}, \ldots, T_{N} u_{1}\right) \in$ gra $T$.

The second proof of Theorem 3.1 employs the classical two-marginal fact that a monotone and continuous mapping is maximally monotone [6, Corollary 20.28], Lemma 2.3 and Theorem 2.5.

Proof. Since $A_{1}(x)=\sum_{i=2}^{N} T_{i}(x)$ for every $x \in H$, by employing Lemma 2.3 it follows that $A_{1}$ is a monotone and continuous mapping, hence, maximally monotone. Consequently, by employing Theorem 2.5 we conclude that $\Gamma$ is maximally monotone.

\section{Maximal $c$-monotonicity of $c$-splitting sets, $c$-conjugate tuples and multi-marginal convex analysis}

We begin our discussion of $c$-splitting tuples by a known observation regarding the subdifferentials of the splitting functions: As in $[12,18,27]$ we observe that if $\left(f_{1}, \ldots, f_{N}\right)$ is a $c$-splitting tuple 
of $\Gamma \subseteq X$, then given $x=\left(x_{1}, \ldots, x_{N}\right) \in \Gamma$ and for any $x_{1}^{\prime} \in X_{1}$,

$$
\sum_{i=1}^{N} f_{i}\left(x_{i}\right)=c\left(x_{1}, \ldots, x_{N}\right)
$$

and $\quad c\left(x_{1}^{\prime}, x_{2}, \ldots, x_{N}\right) \leq f_{1}\left(x_{1}^{\prime}\right)+\sum_{i=2}^{N} f_{i}\left(x_{i}\right)$.

Summing up these two inequalities followed by simplifying, we see that

$$
f_{1}\left(x_{1}\right)+\left\langle x_{1}^{\prime}, x_{2}+\cdots+x_{N}\right\rangle \leq f_{1}\left(x_{1}^{\prime}\right)+\left\langle x_{1}, x_{2}+\cdots+x_{N}\right\rangle, \quad \text { that is, } \sum_{i=2}^{N} x_{i} \in \partial f_{1}\left(x_{1}\right) .
$$

Similarly, we conclude that for each $1 \leq i_{0} \leq N$,

$$
\sum_{i \neq i_{0}} x_{i} \in \partial f_{i_{0}}\left(x_{i_{0}}\right) \text {. }
$$

Since gra $A_{i_{0}}=\left\{\left(x_{i_{0}}, \sum_{i \neq i_{0}} x_{i}\right) \mid\left(x_{1}, \ldots, x_{N}\right) \in \Gamma\right\}$, this implies

$$
\operatorname{gra}\left(A_{i_{0}}\right) \subseteq \operatorname{gra}\left(\partial f_{i_{0}}\right)
$$

Similar observations and $c$-monotonicity properties of $\Gamma$ from Section 2 are also related to the Wasserstein barycenter as can be seen, for example, in [1].

We continue our discussion by a characterization of $c$-splitting tuples and their generated $c$ splitting sets in terms of the Moreau envelopes of the splitting functions.

Theorem 4.1 For each $1 \leq i \leq N$, let $\left.\left.f_{i}: X_{i} \rightarrow\right]-\infty,+\infty\right]$ be proper, lower semicontinuous, and convex. Then $c \leq \bigoplus_{i=1}^{N} f_{i}$ if and only if

$$
\forall s \in H, \quad e_{f_{1}^{*}}(s)+\cdots+e_{f_{N}^{*}}(s) \leq q(s) .
$$

Now assume this is the case, and let $\Gamma \subseteq X$ be the c-splitting set generated by $\left(f_{1}, \ldots, f_{N}\right)$. Then equality in (22) holds if and only if $s=x_{1}+\cdots+x_{N}$ where $\left(x_{1} \ldots, x_{N}\right) \in \Gamma$.

Proof. The inequality $c \leq \bigoplus_{i=1}^{N} f_{i}$ holds if and only if for all $\left(x_{1}, \ldots, x_{N}\right) \in X$,

$$
\begin{aligned}
& c\left(x_{1}, \ldots, x_{N}\right) \leq \sum_{i=1}^{N} f_{i}\left(x_{i}\right) \\
\Leftrightarrow & q\left(x_{1}+\cdots+x_{N}\right)=c\left(x_{1}, \ldots, x_{N}\right)+\sum_{i=1}^{N} q\left(x_{i}\right) \leq \sum_{i=1}^{N}\left(f_{i}\left(x_{i}\right)+q\left(x_{i}\right)\right) .
\end{aligned}
$$

We see that (23) holds with equality only when $\left(x_{1}, \ldots, x_{N}\right) \in \Gamma$ if and only if (24) holds with equality only when $\left(x_{1}, \ldots, x_{N}\right) \in \Gamma$. Let $\varphi: X \rightarrow \mathbb{R}$ be defined by

$$
\varphi\left(x_{1}, \ldots, x_{N}\right)=q\left(x_{1}+\cdots+x_{N}\right) .
$$


Then, using [6, Corollary 15.28(i)], we have

$$
\forall\left(x_{1}, \ldots, x_{N}\right) \in X, \quad \varphi^{*}\left(x_{1}, \ldots, x_{N}\right)=q\left(x_{1}\right)+\iota_{\Delta}\left(x_{1}, \ldots, x_{N}\right) .
$$

Since for each $1 \leq i \leq N,\left(f_{i}+q\right)^{*}=e_{f_{i}^{*}}$ (see, for example, [6, Proposition 14.1]), we arrive at

$$
\left(\bigoplus_{i=1}^{N}\left(f_{i}+q\right)\right)^{*}=\bigoplus_{i=1}^{N}\left(f_{i}+q\right)^{*}=\bigoplus_{i=1}^{N} e_{f_{i}^{*}}
$$

Consequently, (classical) Fenchel conjugation transforms (24) into (22) and vise versa.

We now address the case of equality in (22). Let $\left(x_{1} \ldots, x_{N}\right) \in X$ and $s=x_{1}+\cdots+x_{n}$. Then for each $1 \leq i \leq N$, by the Fenchel-Young inequality,

$$
\left\langle s, x_{i}\right\rangle \leq\left(f_{i}+q\right)^{*}(s)+\left(f_{i}+q\right)\left(x_{i}\right)=e_{f_{i}^{*}}(s)+\left(f_{i}+q\right)\left(x_{i}\right)
$$

with equality if and only if $x_{i} \in \partial\left(f_{i}+q\right)^{*}(s)$, i.e., since $\left(f_{i}+q\right)^{*}=e_{f_{i}^{*}}$ is Fréchet differentiable (see, e.g., [6, Proposition 12.30]), $x_{i}=\nabla e_{f_{i}^{*}}(s)$. By summing up (25) over $i$, we obtain

$$
\langle s, s\rangle=\sum_{i=1}^{N}\left\langle s, x_{i}\right\rangle \leq \sum_{i=1}^{N}\left(e_{f_{i}^{*}}(s)+\left(f_{i}+q\right)\left(x_{i}\right)\right)
$$

with equality if and only if $x_{i}=\nabla e_{f_{i}^{*}}(s)$ for every $1 \leq i \leq N$.

$(\Leftarrow)$ : Suppose that $x=\left(x_{1}, \ldots, x_{N}\right)$ is in the $c$-splitting set $\Gamma$ generated by $\left(f_{1}, \ldots, f_{N}\right)$ and set $s=S(x)$. We prove equality in (22). It follows from (20) that for each $1 \leq i \leq N$,

$$
\sum_{j \neq i} x_{j}=s-x_{i} \in \partial f_{i}\left(x_{i}\right) \quad \Leftrightarrow \quad s \in \partial\left(f_{i}+q\right)\left(x_{i}\right),
$$

which, in turn, implies that $x_{i} \in \partial\left(f_{i}+q\right)^{*}(s)$, that is, $x_{i}=\nabla e_{f_{i}^{*}}(s)$. Since in this case there is equality in (26) and in (24), we obtain equality in (22).

$(\Rightarrow)$ : Let $s \in H$ be a point where equality in (22) holds. Since $\sum_{i=1}^{N} e_{f_{i}^{*}}$ and $q$ are Fréchet differentiable and $\sum_{i=1}^{N} e_{f_{i}^{*}} \leq q$, then at the point of equality $s$ we have

$$
\nabla\left(\sum_{i=1}^{N} e_{f_{i}^{*}}\right)(s)=\nabla q(s)=s .
$$

For each $1 \leq i \leq N$, set $x_{i}=\operatorname{Prox}_{f_{i}}(s)=\nabla e_{f_{i}^{*}}(s)$ (see, e.g., [6, eq (14.7)]). Then it follows that $s=x_{1}+\cdots+x_{N}$. Thus, in order to complete the proof it is enough to prove that $\left(x_{1}, \ldots, x_{N}\right) \in$ $\Gamma$ or, equivalently, that there is equality in (24). Indeed, Moreau's decomposition (see, e.g., [6, Remark 14.4]) implies that $e_{f_{i}}+e_{f_{i}^{*}}=q$ for each $1 \leq i \leq N$. Consequently,

$$
\sum_{i=1}^{N} e_{f_{i}^{*}}(s)=q(s) \quad \text { is equivalent to } \quad \sum_{i=1}^{N} e_{f_{i}}(s)=(N-1) q(s) .
$$


We also note that for each $1 \leq i \leq N, x_{i}=\operatorname{Prox}_{f_{i}}(s)$ implies that

$$
e_{f_{i}}(s)=\min _{x \in H}\left(f_{i}(x)+q(s-x)\right)=f_{i}\left(x_{i}\right)+q\left(s-x_{i}\right) .
$$

Thus, we arrive at

$$
\begin{aligned}
& \sum_{i=1}^{N}\left(q\left(s-x_{i}\right)+f_{i}\left(x_{i}\right)\right)=(N-1) q(s) \\
\Leftrightarrow \quad & -\sum_{i=1}^{N}\left\langle s, x_{i}\right\rangle+\sum_{i=1}^{N}\left(f_{i}\left(x_{i}\right)+q\left(x_{i}\right)\right)=-q(s) \\
\Leftrightarrow & \sum_{i=1}^{N}\left(f_{i}\left(x_{i}\right)+q\left(x_{i}\right)\right)=q(s) .
\end{aligned}
$$

We now address $c$-conjugation.

Definition 4.2 (c-conjugate tuple) For each $1 \leq i \leq N$, let $\left.\left.f_{i}: X_{i} \rightarrow\right]-\infty,+\infty\right]$ be a proper function. We say that $\left(f_{1}, \ldots, f_{N}\right)$ is a c-conjugate tuple if for each $1 \leq i_{0} \leq N$,

$$
f_{i_{0}}\left(x_{i_{0}}\right)=\left(\bigoplus_{i \neq i_{0}} f_{i}\right)^{c}\left(x_{i_{0}}\right):=\sup _{i \neq i_{0}, x_{i} \in X_{i}} c\left(x_{1}, \ldots, x_{i_{0}}, \ldots, x_{N}\right)-\sum_{i \neq i_{0}} f_{i}\left(x_{i}\right), \quad x_{i_{0}} \in X_{i_{0}} .
$$

It follows that if $\left(f_{1}, \ldots, f_{N}\right)$ is a $c$-conjugate tuple, then $f_{i}$ is lower semicontinuous and convex for each $1 \leq i \leq N$. Furthermore, it is known (see [12] and [10]) that given a $c$-splitting tuple $\left(u_{1}, \ldots, u_{N}\right)$ of a set $\Gamma \subseteq X$, it can be relaxed into a $c$-conjugate $c$-splitting tuple $\left(f_{1}, \ldots, f_{N}\right)$ of $\Gamma$ by setting

$$
f_{1}=\left(\bigoplus_{2 \leq i \leq N} u_{i}\right)^{c}
$$

inductively,

$$
f_{i_{0}}=\left(\bigoplus_{1 \leq i \leq i_{0}-1} f_{i} \oplus \bigoplus_{i_{0}+1 \leq i \leq N} u_{i}\right)^{c} \quad \text { for } 2 \leq i_{0} \leq N-1
$$

and finally

$$
f_{N}=\left(\bigoplus_{1 \leq i \leq N-1} f_{i}\right)^{c}
$$

In the case $N=2$, let $\left.\left.f_{1}: X_{1} \rightarrow\right]-\infty,+\infty\right]$ be proper, lower semicontinuous and convex, let $\left.\left.f_{2}=f_{1}^{*}: X_{2} \rightarrow\right]-\infty,+\infty\right]$ be its conjugate and let $\Gamma=\operatorname{gra}\left(\partial f_{1}\right) \subseteq X_{1} \times X_{2}$. Then it is well known that $\Gamma$ is maximally monotone, see, e.g., [6, Theorem 20.25]. Since $f_{1}=f_{1}^{* *}=f_{2}^{c}$ and also $f_{2}=f_{1}^{c}$, then we can restate as follows: 
Let $\Gamma \subseteq X_{1} \times X_{2}$ be the $c$-splitting set generated by the $c$-conjugate pair $\left(f_{1}, f_{2}\right)$. Then $\Gamma$ is maximally $c$-monotone and determines its $c$-conjugate $c$-splitting tuple $\left(f_{1}, f_{2}\right)$ uniquely up to an additive constant pair $(\rho,-\rho)$ with $\rho \in \mathbb{R}$.

A generalization to an arbitrary $N \geq 2$ would be

Let $\Gamma \subseteq X$ be the $c$-splitting set generated by the $c$-conjugate tuple $\left(f_{1}, \ldots, f_{N}\right)$. Then $\Gamma$ is maximally $c$-monotone and determines its $c$-conjugate $c$-splitting tuple $\left(f_{1}, \ldots, f_{N}\right)$ uniquely up to an additive constant tuple $\left(\rho_{1}, \ldots, \rho_{N}\right)$ such that $\sum_{i=1}^{N} \rho_{i}=0$.

To the best of our knowledge, whether or not this latter assertion is true in general is still open. We do, however, provide a positive answer in a more particular case in Theorem 4.6 and additional insight in Theorem 4.3.

Furthermore, we note that in the case $N=2$, given a conjugate pair $\left(f_{1}, f_{2}\right)$, Moreau's decomposition can be restated as

$$
e_{f_{1}^{*}}+e_{f_{2}^{*}}=q \quad \text { and } \quad \operatorname{Prox}_{f_{1}}+\operatorname{Prox}_{f_{2}}=\mathrm{Id} .
$$

Combining our discussion with Theorems 4.1 and 2.3, we arrive at the following generalized multi-marginal convex analytic assertions which, in particular, generalize the decomposition (28). To this end, we again recall that for each $1 \leq i_{0} \leq N$,

$$
\operatorname{gra} A_{i_{0}}=\left\{\left(x_{i_{0}}, \sum_{i \neq i_{0}} x_{i}\right) \mid\left(x_{1}, \ldots, x_{N}\right) \in \Gamma\right\} \text {. }
$$

Theorem 4.3 For each $1 \leq i \leq N$, let $\left.\left.f_{i}: X_{i} \rightarrow\right]-\infty,+\infty\right]$ be convex, lower semicontinuous, and proper. Suppose that $\Gamma \subseteq X$ is the c-splitting set generated by $\left(f_{1}, \ldots, f_{N}\right)$. Then the following assertions are equivalent:

(i) There exist $1 \leq i_{0} \leq N$ such that $A_{i_{0}}$ is maximally monotone;

(ii) There exist $1 \leq i_{0} \leq N$ such that $A_{i_{0}}=\partial f_{i_{0}}$;

(iii) $A_{i}=\partial f_{i}$ for each $1 \leq i \leq N$;

(iv) $\operatorname{Prox}_{f_{1}}+\cdots+\operatorname{Prox}_{f_{N}}=\mathrm{Id}$;

(v) $e_{f_{1}^{*}}+\cdots+e_{f_{N}^{*}}=q$.

In this case

(A) $\Gamma$ is maximally c-monotone (and, consequently, maximally c-cyclically monotone); 
(B) $\left(f_{1}, \ldots, f_{N}\right)$ is a c-conjugate c-splitting tuple of $\Gamma$. Moreover, $\Gamma$ determines its c-conjugate c-splitting tuple $\left(f_{1}, \ldots, f_{N}\right)$ uniquely up to an additive constant tuple $\left(\rho_{1}, \ldots, \rho_{N}\right)$ such that $\sum_{i=1}^{N} \rho_{i}=0$.

Proof. (i) $\Rightarrow$ (ii): $\partial f_{i_{0}}$ is monotone and gra $\left(A_{i_{0}}\right) \subseteq \operatorname{gra}\left(\partial f_{i_{0}}\right)$ (see (21)). Consequently, since $A_{i_{0}}$ is maximally monotone, it follows that $A_{i_{0}}=\partial f_{i_{0}}$.

(ii) $\Rightarrow$ (iii): $A_{i_{0}}=\partial f_{i_{0}}$ is maximally monotone as the subdifferential of a proper lower semicontinuous convex function. Consequently, it follows from Theorem 2.5(i)\&(ii) that $A_{i}$ is maximally monotone for each $1 \leq i \leq N$. Now, $\partial f_{i}$ is monotone and gra $\left(A_{i}\right) \subseteq \operatorname{gra}\left(\partial f_{i}\right)$ (see (21)). Consequently, since $A_{i}$ is maximally monotone, it follows that $A_{i}=\partial f_{i}$.

(iii) $\Rightarrow$ (iv): Follows from Theorem 2.5(i)\&(iv) since $A_{i}=\partial f_{i}$ is maximally monotone and $\operatorname{Prox} f_{i}=J_{\partial f_{i}}=J_{A_{i}}$.

(iv) $\Rightarrow(v)$ : By integrating (iv) we obtain the equality in (v) up to an additive constant. Theorem 4.1 implies that equality in $(\mathrm{v})$ holds on $S(\Gamma)$; thus, the additive constant vanishes.

(v) $\Rightarrow$ (i): By Theorem 4.1 equality in (v) holds only on $S(\Gamma)$. Consequently, (v) implies that $S(\Gamma)=H$. By employing Theorem 2.5(vi)\&(i), we obtain (i).

In this case Theorem 2.5 also implies $\Gamma$ is maximally $c$-monotone. Thus, it remains to prove (B). By our preliminary discussion there exists a $c$-conjugate $c$-splitting tuple $\left(h_{1}, \ldots, h_{N}\right)$ of $\Gamma$. From (iii) and from (21) we conclude that $\operatorname{gra}\left(\partial f_{i}\right)=\operatorname{gra}\left(A_{i}\right) \subseteq \operatorname{gra}\left(\partial h_{i}\right)$ which, by maximality, implies that $\partial f_{i}=\partial h_{i}$ for each $1 \leq i \leq N$. Here there exists a constant tuple $\left(\rho_{1}, \ldots, \rho_{N}\right) \in \mathbb{R}^{N}$ such that $\left(f_{1}, \ldots, f_{N}\right)=\left(h_{1}, \ldots, h_{N}\right)+\left(\rho_{1}, \ldots, \rho_{N}\right)$. For $\left(x_{1}, \ldots, x_{N}\right) \in \Gamma$ the equality $\sum_{i=1}^{N} f_{i}\left(x_{i}\right)=$ $\sum_{i=1}^{N} h_{i}\left(x_{i}\right)$ implies that $\sum_{i=1}^{N} \rho_{i}=0$. Consequently, the fact that for each $1 \leq i_{0} \leq N$

$$
f_{i_{0}}-\rho_{i_{0}}=\left(\bigoplus_{i \neq i_{0}}^{N}\left(f_{i}-\rho_{i}\right)\right)^{c}
$$

implies that $\left(f_{1}, \ldots, f_{N}\right)$ is a $c$-conjugate tuple.

We now provide a smoothness criteria in the 3-marginal case where Theorem 4.3(i)-(v)\&(B) are equivalent and imply maximal $c$-monotonicity. To this end we will employ the following facts.

Fact 4.4 ([6, Theorem 14.19]) Let $g: H \rightarrow]-\infty,+\infty]$ be proper, let $h: H \rightarrow]-\infty,+\infty]$ be proper, lower semicontinuous and convex. Set

$$
f: H \rightarrow[-\infty,+\infty]: x \mapsto \begin{cases}g(x)-h(x), & x \in \operatorname{dom}(g) \\ +\infty, & x \notin \operatorname{dom}(g) .\end{cases}
$$

Then

$$
f^{*}(y)=\sup _{v \in \operatorname{dom}\left(h^{*}\right)}\left(g^{*}(y+v)-h^{*}(v)\right) .
$$

Fact 4.5 ([29, Corollary 2.3]) Let $f: \mathbb{R}^{n} \rightarrow \mathbb{R}$ be proper and lower semicontinuous. If $f^{*}$ is essentially smooth, then $f$ is convex. 
Theorem 4.6 Let $n \in \mathbb{N}, N=3$ and $H=\mathbb{R}^{n}$. Let $\left.\left.g: X_{2} \rightarrow\right]-\infty,+\infty\right]$ and $\left.\left.h: X_{3} \rightarrow\right]-\infty,+\infty\right]$ be proper, lower semicontinuous and convex functions. Suppose that $f=(g \oplus h)^{c}$ (in particular if $(f, g, h)$ is a c-conjugate triple) and that $f$ is essentially smooth. Let $\Gamma$ be the c-splitting set generated by $(f, g, h)$. Then assertions (i)-(v) of Theorem 4.3 hold and $\Gamma$ is maximally c-monotone.

Proof. Since $f=(g \oplus h)^{c}$ and $\operatorname{dom}(g+q)^{*}=\operatorname{dom}\left(e_{g^{*}}\right)=\mathbb{R}^{n}$, then by employing Fact 4.4 in (29) and then Moreau's decomposition in (30) we see that

$$
\begin{aligned}
(f+q)(x) & =\sup _{y, z \in \mathbb{R}^{n}}(c(x, y, z)-g(y)-h(z)+q(x)) \\
& =\sup _{y, z \in \mathbb{R}^{n}}(\langle x, y\rangle+\langle y, z\rangle+\langle z, x\rangle+q(x)-g(y)-h(z)) \\
& =\sup _{y \in \mathbb{R}^{n}}\left(\langle x, y\rangle+h^{*}(x+y)+q(x)-g(y)\right) \\
& =\sup _{y \in \mathbb{R}^{n}}\left(h^{*}(x+y)+q(x+y)-(g(y)+q(y))\right) \\
& =\left(\left(h^{*}+q\right)^{*}-(g+q)^{*}\right)^{*}(x) \\
& =\left(e_{h}-e_{g^{*}}\right)^{*}(x)=\left(q-e_{g^{*}}-e_{h^{*}}\right)^{*}(x) .
\end{aligned}
$$

Since $f+q$ is essentially smooth, Fact 4.5 implies that $q-e_{g^{*}}-e_{h^{*}}$ is convex. Consequently,

$$
e_{f^{*}}=(f+q)^{*}=\left(q-e_{g^{*}}-e_{h^{*}}\right)^{* *}=q-e_{g^{*}}-e_{h^{*}}
$$

that is, $e_{f^{*}}+e_{g^{*}}+e_{h^{*}}=q$.

Remark 4.7 In our discussion in the last paragraph of Section 2 we pointed out that in the partition of the identity in Theorem 2.5(iv) any partial sum of the firmly nonexpansive mappings is again firmly nonexpansive and, furthermore, that general partitions of the identity into firmly nonexpansive mappings partial sums may fail to be firmly nonexpansive. Thus, in the context of $c$-splitting sets a natural question is: Given a partition of the identity into proximal mappings, are partial sums also proximal mappings? Unlike general firmly nonexpansive mappings, a positive answer to this question is provided by [4, Theorem 4.2].

\section{Examples, observations and remarks}

We now apply our results in order to determine maximality of $c$-monotone sets. Given a multimarginal $c$-cyclically monotone set $\Gamma \subseteq X$, the problem of constructing a $c$-splitting tuple is, in general, nontrivial. Nevertheless, constructions which are independent of maximality and uniqueness considerations are available for some classes of $c$-cyclically monotone sets (for example, see [5] for the case $N \geq 3$ ). We also note that $c$-splitting tuples can be constructed via (21) if it is known, in addition, that the antiderivatives $f_{i}$ are unique up to additive constants, as guaranteed by Theorem 4.3. Now, suppose that a $c$-splitting tuple is already given. The computation and classification 
of the $c$-splitting tuple as being a c-conjugate tuple were, thus far, nontrivial. We employ our results for such classifications in the following examples. For these cases, we are able to conclude $c$-conjugacy without additional nontrivial computations of multi-marginal conjugates. In addition, we demonstrate finer aspects of multi-marginal maximal monotonicity.

Example 5.1 For each $1 \leq i \leq N$, set $X_{i}=\mathbb{R}^{d}$ and let $Q_{i} \in \mathbb{R}^{d \times d}$ be symmetric, positive definite, and pairwise commuting. Set

$$
\Gamma=\left\{\left(Q_{1} v, \ldots, Q_{N} v\right) \mid v \in \mathbb{R}^{d}\right\} .
$$

For each $1 \leq i \leq M$, define $M_{i} \in \mathbb{R}^{d \times d}$ by

$$
M_{i}=\left(\sum_{k \neq i} Q_{k}\right) Q_{i}^{-1}
$$

In [5, Example 3.4], it was established that

$$
\forall\left(x_{1}, \ldots, x_{N}\right) \in X, \quad c\left(x_{1}, \ldots, x_{N}\right)=\sum_{1 \leq i<j \leq N}\left\langle x_{i}, x_{j}\right\rangle \leq \sum_{1 \leq i \leq N} q_{M_{i}}\left(x_{i}\right),
$$

where $q_{M_{i}}(x)=\frac{1}{2}\left\langle x, M_{i} x\right\rangle$, and equality holds if and only if $\left(x_{1}, \ldots, x_{N}\right) \in \Gamma$.

Thus, we conclude that $\Gamma$ is the $c$-splitting set generated by the tuple $\left(q_{M_{1}}, \ldots, q_{M_{N}}\right)$, and that $A_{i}=M_{i}=\nabla q_{M_{i}}$ for each $1 \leq i \leq N$. Consequently, Theorem 4.3 implies that $\left(q_{M_{1}}, \ldots, q_{M_{N}}\right)$ is a $c$-conjugate $c$-splitting tuple of $\Gamma$, and that $\Gamma$ is maximally $c$-monotone.

The maximal $c$-monotonicity of $\Gamma$ is also implied by Theorem 3.1 via continuity of a parametrization, say,

$$
\Gamma=\left\{\left(v, Q_{2} Q_{1}^{-1} v \ldots, Q_{N} Q_{1}^{-1} v\right) \mid v \in \mathbb{R}^{d}\right\} .
$$

As a simple application of Example 5.1, we now generalize the well-known classical fact that the only conjugate pair of the form $(f, f)$ is $(f, f)=(q, q)$ and that in this case the generated splitting set is the graph of the identity mapping.

Corollary 5.2 (self $c$-conjugate tuple) The only c-conjugate tuple of the form $(f, \ldots, f)$ is

$$
(f, \ldots, f)=(N-1)(q, \ldots, q) \text {. }
$$

In this case, the generated c-splitting set is $\Gamma=\Delta$.

Proof. In the settings of Example 5.1 we let $Q_{i}=\operatorname{Id}$ for each $1 \leq i \leq N$. Then $\Gamma=\Delta$ and $q_{M_{i}}=(N-1) q$ for each $1 \leq i \leq N$. We conclude that $(N-1)(q, \ldots, q)$ is a $c$-conjugate $c$-splitting tuple and generates the $c$-splitting set $\Delta$. We now prove that it is the only $c$-conjugate tuple of this form. Let $(f, \ldots, f)$ be a $c$-conjugate tuple. Then for $1 \leq i_{0} \leq N$ and for $x_{i_{0}} \in X_{i_{0}}$,

$$
f\left(x_{i_{0}}\right)=\sup _{i \neq i_{0}, x_{i} \in H}\left(c\left(x_{1}, \ldots, x_{i_{0}}, \ldots, x_{N}\right)-\sum_{i \neq i_{0}} f\left(x_{i}\right)\right) .
$$


By letting $x_{i}=x_{i_{0}}$ for every $i$ in the supremum in (31) we see that

$$
f\left(x_{i_{0}}\right) \geq c\left(x_{i_{0}}, \ldots, x_{i_{0}}\right)-(N-1) f\left(x_{i_{0}}\right) \quad \Rightarrow \quad N f \geq N(N-1) q \quad \Rightarrow \quad f \geq(N-1) q .
$$

Consequently,

$$
f=\left(\bigoplus_{i \neq i_{0}} f\right)^{c} \leq\left(\bigoplus_{i \neq i_{0}} q\right)^{c}=(N-1) q
$$

A similar type of construction to the one of Example 5.1, however, a nonlinear one, is available when the marginals are one-dimensional.

Example 5.3 For each $1 \leq i \leq N$, let $\alpha_{i}: \mathbb{R} \rightarrow \mathbb{R}$ be a continuous, strictly increasing and surjective function with $\alpha_{i}(0)=0$. Let $\Gamma$ be the curve in $\mathbb{R}^{N}$ defined by

$$
\Gamma=\left\{\left(\alpha_{1}(t), \ldots, \alpha_{N}(t)\right) \mid t \in \mathbb{R}\right\}
$$

and for each $1 \leq i \leq N$, let

$$
f_{i}\left(x_{i}\right)=\int_{0}^{x_{i}}\left(\sum_{k \neq i} \alpha_{k}\left(\alpha_{i}^{-1}(t)\right)\right) d t .
$$

In [5, Example 4.3], it was established that

$$
\sum_{1 \leq i<j \leq N} x_{i} x_{j} \leq \sum_{i=1}^{N} \int_{0}^{x_{i}}\left(\sum_{k \neq i} \alpha_{k}\left(\alpha_{i}^{-1}(t)\right)\right) d t \quad \forall\left(x_{1}, \ldots, x_{N}\right) \in \mathbb{R}^{N}
$$

and that equality in (33) holds if and only if $x_{j}=\alpha_{j}\left(\alpha_{i}^{-1}\left(x_{i}\right)\right)$ for every $1 \leq i<j \leq N$, namely, if $\left(x_{1}, \ldots, x_{N}\right) \in \Gamma$. We now conclude that $\Gamma$ is the $c$-splitting set generated by the tuple $\left(f_{1}, \ldots, f_{N}\right)$ and that for each $1 \leq i \leq N$,

$$
A_{i}=\nabla f_{i}=\sum_{k \neq i} \alpha_{k} \circ \alpha_{i}^{-1}
$$

Consequently, Theorem 4.3 implies that $\left(f_{1}, \ldots, f_{n}\right)$ is a $c$-conjugate $c$-splitting tuple of the maximally $c$-monotone curve $\Gamma$. Similar to Example 5.1, the maximal $c$-monotonicity of $\Gamma$ can also be deduced via continuity.

A linear example of a different type, where none of the two marginal projections of $\Gamma$ is monotone, but where, however, $\Gamma$ is $c$-cyclically monotone, is available for $N=3$ and 2-dimensional marginals.

Example 5.4 Suppose that $N=3$ and that $X_{1}=X_{2}=X_{3}=\mathbb{R}^{2}$. We set

$$
M_{1}=2\left(\begin{array}{ll}
1 & 0 \\
0 & 0
\end{array}\right), \quad M_{2}=2\left(\begin{array}{ll}
1 & 0 \\
0 & 1
\end{array}\right), \quad M_{3}=\frac{1}{7}\left(\begin{array}{ll}
8 & 3 \\
3 & 2
\end{array}\right)
$$


and

$$
\Delta_{2}=\{(a, a) \mid a \in \mathbb{R}\} \subseteq \mathbb{R}^{2}
$$

Set

$$
f_{1}=\iota_{\mathbb{R} \times\{0\}}+q_{M_{1}}, \quad f_{2}=\iota_{\Delta_{2}}+q_{M_{2}}=\iota_{\Delta_{2}}+2 q, \quad \text { and } \quad f_{3}=q_{M_{3}} .
$$

Furthermore, set $v_{1}=((0,0),(-1,-1),(1,-5)), v_{2}=((1,0),(2,2),(0,7))$ and

$$
\Gamma=\operatorname{span}\left\{v_{1}, v_{2}\right\}=\{((s, 0),(2 s-t, 2 s-t),(t, 7 s-5 t)) \mid s, t \in \mathbb{R}\} .
$$

It was established in [5, Example 3.5] that

$$
\left\langle x_{1}, x_{2}\right\rangle+\left\langle x_{2}, x_{3}\right\rangle+\left\langle x_{3}, x_{1}\right\rangle \leq f_{1}\left(x_{1}\right)+f_{2}\left(x_{2}\right)+f_{3}\left(x_{3}\right) \quad \text { for all } \quad\left(x_{1}, x_{2}, x_{3}\right) \in\left(\mathbb{R}^{2}\right)^{3}
$$

with equality if and only if $\left(x_{1}, x_{2}, x_{3}\right) \in \Gamma$, namely, $\Gamma$ is the $c$-splitting set generated by the tuple $\left(f_{1}, f_{2}, f_{3}\right)$ and that none of the two marginal projections $\Gamma_{1,2}, \Gamma_{1,3}$ and $\Gamma_{2,3}$ of $\Gamma$, is monotone.

We observe that the matrix representation of the mapping

$$
(t, 7 s-5 t) \mapsto(s, 0)+(2 s-t, 2 s-t) \quad s, t \in \mathbb{R}
$$

is $M_{3}$. Consequently, we see that $A_{3}=M_{3}=\nabla f_{3}$. Thus, by employing Theorem 4.3 we conclude that $\left(f_{1}, f_{2}, f_{3}\right)$ is a $c$-conjugate $c$-splitting tuple of the maximally $c$-monotone subspace $\Gamma$ of $\left(\mathbb{R}^{2}\right)^{3}$.

In all of our examples thus far, the set $\Gamma$ was a maximally $c$-monotone $c$-splitting set. We now present maximally $c$-monotone sets which are not $c$-splitting sets. To this end, we note the following simple fact: Suppose that the set $\Gamma \subseteq X$ is $n$-c-monotone, then for each $1 \leq i_{0} \leq N$ the mapping $A_{i_{0}}: H \rightrightarrows H$ is $n$-monotone. Indeed, let $\Gamma$ be $n$-c-monotone and assume, without the loss of generality, that $i_{0}=1$. Let $\left(x_{1}^{1}, \ldots, x_{N}^{1}\right), \ldots,\left(x_{1}^{n}, \ldots, x_{N}^{n}\right) \in \Gamma$ and $\sigma \in S_{n}$. Then a straightforward computation implies that the inequality

$$
\sum_{j=1}^{n} c\left(x_{1}^{j}, x_{2}^{\sigma(j)}, \ldots, x_{N}^{\sigma(j)}\right) \leq \sum_{j=1}^{n} c\left(x_{1}^{j}, \ldots, x_{N}^{j}\right)
$$

leads to the inequality

$$
\sum_{j=1}^{n}\left\langle x_{1}^{j}, \sum_{i=2}^{N} x_{i}^{\sigma(j)}\right\rangle \leq \sum_{j=1}^{n}\left\langle x_{1}^{j}, \sum_{i=2}^{N} x_{i}^{j}\right\rangle .
$$

Thus, we see that if $\Gamma$ is $n-c$-monotone, then $A_{1}$ is $n$-monotone. To sum up,

if for some $1 \leq i_{0} \leq N$ the mapping $A_{i_{0}}$ is not cyclically monotone, then the set $\Gamma$ is not a $c$-splitting set. 
Indeed, otherwise, $\Gamma$ would have been $c$-cyclically monotone (as we recollected after Definition 1.2) and, by the above argument, for all $1 \leq i_{0} \leq N$ the mapping $A_{i_{0}}$ would have been cyclically monotone.

We now address a trivial embedding of all classical maximally monotone operators in the multimarginal framework. In particular, we obtain maximally $c$-monotone mappings which are not c-cyclically monotone.

Example 5.5 Let $A: H \rightrightarrows H$ be a maximally monotone mapping. We set $\Gamma \subseteq X$ by

$$
\Gamma=\left\{\left(x_{1}, x_{2}, 0, \ldots, 0\right) \mid x_{2} \in A x_{1}\right\} \text {. }
$$

Then $\Gamma$ is $c$-monotone and we see that $A_{1}=A$ is maximally monotone. Consequently, by invoking Theorem 2.5 (ii) we conclude that $\Gamma$ is maximally $c$-monotone. In addition, we see that $A$ is $n$ monotone if and only if $\Gamma$ is $n$-c-monotone. Therefore, if $A$ is not $n$-monotone for some $n \geq 3$, then $\Gamma$ is not $n-c$-monotone. Furthermore, since the $n$-c-monotonicity of a set is invariant under shifts, the set $\Gamma=\left\{\left(x_{1}, x_{2}, \rho_{3}, \ldots, \rho_{N}\right) \mid x_{2} \in A x_{1}\right\}$ is also maximally monotone for any constant vectors $\rho_{3}, \ldots, \rho_{N} \in H$.

Our next example of a maximally $c$-monotone set which is not a $c$-splitting set does not follow from an embedding of the type in Example 5.5.

Example 5.6 Set $N=3$ and for each $1 \leq i \leq 3$ set $X_{i}=\mathbb{R}^{2}$. Let $R_{\theta}$ denote the counterclockwise rotation by the angle $\theta$ in $\mathbb{R}^{2}$. Let the set $\Gamma \subseteq X=\left(\mathbb{R}^{2}\right)^{3}$ be defined by

$$
\Gamma=\left\{\left(x, \frac{\sqrt{3}}{2} R_{-\pi / 2} x, \frac{\sqrt{3}}{2} R_{-\pi / 2} x\right) \mid x \in \mathbb{R}^{2}\right\} .
$$

It follows that

$$
\operatorname{gra} A_{1}=\left\{\left(x, \sqrt{3} R_{-\pi / 2} x\right) \mid x \in \mathbb{R}^{2}\right\} \quad \Longrightarrow \quad A_{1}=\sqrt{3} R_{-\pi / 2}
$$

Since $\Gamma=\left\{\left(\frac{2}{\sqrt{3}} R_{\pi / 2} x, x, x\right) \mid x \in \mathbb{R}^{2}\right\}$, we have

$$
\operatorname{gra} A_{2}=\operatorname{gra} A_{3}=\left\{\left(x, x+\frac{2}{\sqrt{3}} R_{\pi / 2} x\right) \mid x \in \mathbb{R}^{2}\right\} \quad \Longrightarrow \quad A_{2}=A_{3}=\sqrt{\frac{7}{3}} R_{\arctan (2 / \sqrt{3})} .
$$

We see that $A_{1}, A_{2}$, and $A_{3}$ are maximally monotone. Consequently, for each $\varnothing \neq K \subsetneq\{1,2,3\}$, the mapping $A_{K}$ is maximally monotone and it now follows from Theorem 2.5 that $\Gamma$ is maximally $c$-monotone in $X$. Furthermore, since $A_{1}$ is not 3-c-cyclically monotone, it is not $c$-cyclically monotone and, consequently, $\Gamma$ is not a $c$-splitting set. By a straightforward computation, it follows that

$$
J_{A_{1}}=\frac{1}{2} R_{\pi / 3}, \quad J_{A_{2}}=J_{A_{3}}=\frac{\sqrt{3}}{4} R_{-\pi / 6} \quad \text { and } \quad J_{A_{1}}+J_{A_{2}}+J_{A_{3}}=\mathrm{Id} .
$$

Finally, from (34) it is easy to see that $\Gamma_{i, j}$ is monotone for all $1 \leq i<j \leq 3$. 
We see that in the case $N=3$ the set $\Gamma$ is $c$-monotone if and only if the mappings $A_{1}, A_{2}$ and $A_{3}$ are monotone. In the following example we demonstrate that the monotonicity of all of the $A_{i}{ }^{\prime}$ s no longer implies the $c$-monotonicity of $\Gamma$ in the case when $N \geq 4$.

Example 5.7 In [4, Lemma 4.2 and Example 4.3] it was established that: In $X=\mathbb{R}^{2}$, let $n \in$ $\{2,3, \ldots\}$, let $\theta \in] \arccos (1 / \sqrt{2}), \arccos (1 / \sqrt{2 n})]$, set $\alpha=1 /(2 n \cos (\theta))$, and denote by $R_{\theta}$ the counterclockwise rotator by $\theta$. Then the following hold:

(i) $\alpha R_{\theta}$ and $\alpha R_{-\theta}$ are firmly nonexpansive.

(ii) $n \alpha R_{\theta}$ and $n \alpha R_{-\theta}$ are not firmly nonexpansive.

(iii) $n \alpha R_{\theta}+n \alpha R_{-\theta}=$ Id.

We employ these facts to construct a set $\Gamma$ as follows. We set $N=2 n$ and

$$
T_{i}= \begin{cases}\alpha R_{\theta}, & 1 \leq i \leq n \\ \alpha R_{-\theta}, & n+1 \leq i \leq 2 n\end{cases}
$$

Define

$$
\Gamma=\left\{\left(T_{1} x, \ldots, T_{2 n} x\right) \mid x \in \mathbb{R}^{2}\right\} \subseteq X=\left(\mathbb{R}^{2}\right)^{2 n} .
$$

It then follows that for each $1 \leq i \leq N$, the mapping $J_{A_{i}}=T_{i}$ is firmly nonexpansive with full domain. We conclude that the set $\Gamma$ possesses the following properties:

(iv) for each $1 \leq i \leq N$, the mapping $A_{i}$ is maximally monotone,

(v) $J_{A_{1}}+\cdots+J_{A_{N}}=$ Id.

However, due to (ii), the mappings

$$
J_{A_{\{1, \ldots, n\}}}=\sum_{i=1}^{n} J_{A_{i}}=\sum_{i=1}^{n} T_{i}=n \alpha R_{\theta}, \quad \text { and similarly } \quad J_{A_{\{n+1, \ldots, 2 n\}}}=n \alpha R_{-\theta}
$$

are not firmly nonexpansive, equivalently, $A_{\{1, \ldots, n\}}=R_{-2 \theta}$ and $A_{\{n+1, \ldots, 2 n\}}=R_{2 \theta}$ are not monotone. Consequently, by employing Lemma 2.3 we conclude that despite the fact that $\Gamma$ possesses properties (iv) and (v), it is not a $c$-monotone set.

Remark 5.8 In [5] the two marginal projections $\Gamma_{i, j}$ of a set $\Gamma \subseteq X$ were employed, it was established that if the $\Gamma_{i, j}$ 's are cyclically monotone, then $\Gamma$ is $c$-cyclically monotone and an explicit construction of a $c$-splitting tuple is provided. However, it was also established that this is a sufficient condition for $c$-cyclic monotonicity of $\Gamma$ but not a necessary one, in general, as can be seen in Example 5.4 where we provide a maximally $c$-cyclically monotone set such that all of its two-marginal projections are not monotone. In the one dimensional case (i.e., $X_{i}=\mathbb{R}$ for each $1 \leq i \leq N)$, it was established that $\Gamma$ is $c$-monotone if and only if all of its two marginal projections 
$\Gamma_{i, j}$ are monotone. With the exception of Example 5.4, in all of our examples of $c$-monotone sets in this section the set $\Gamma$ had monotone two-marginal projections $\Gamma_{i, j}$. Thus, a natural question is: How does the monotonicity and maximal monotonicity of the two-marginal projections $\Gamma_{i, j}$ relate to the c-monotonicity and maximal c-monotonicity of $\Gamma$ ?

Proposition 5.9 Let $X_{i}=\mathbb{R}^{d}$ for each $1 \leq i \leq N$. Let $\Gamma \subseteq X$ be a set. Suppose that for each $1 \leq i<j \leq$ $N$ the set $\Gamma_{i, j}$ is monotone. Then $\Gamma$ is c-monotone.

Proof. The mapping $A_{K}$ is monotone if and only if for every $\left(x_{1}, \ldots, x_{N}\right),\left(y_{1}, \ldots, y_{N}\right) \in \Gamma$,

$$
0 \leq\left\langle\sum_{i \in K} x_{i}-y_{i}, \sum_{j \notin K} x_{j}-y_{j}\right\rangle .
$$

Since the right-hand side is equal to $\sum_{\substack{i \in K \\ j \notin K}}\left\langle x_{i}-y_{i}, x_{j}-y_{j}\right\rangle$ and since, by the monotonicity of $\Gamma_{i, j}$, $0 \leq\left\langle x_{i}-y_{i}, x_{j}-y_{j}\right\rangle$, we see that $A_{K}$ is monotone.

To the best of our knowledge, the question whether the maximal monotonicity of the $\Gamma_{i, j}$ 's implies the maximal $c$-monotonicity of $\Gamma$ is still open.

Finally, we note that the maximal $c$-monotonicity of $\Gamma$ does not imply the maximal monotonicity of the $\Gamma_{i, j}$ 's even when the $\Gamma_{i, j}$ 's are monotone. Indeed, in Example 5.5, we see that although $\Gamma$ is maximally c-monotone, $\Gamma_{i, j}$ is a singleton for all $3 \leq i<j \leq N$, thus $\Gamma_{i, j}$ is monotone but not maximally monotone. Even in the case $N=3, \Gamma_{1,3}$ is a proper subset of the graph of the zero mapping whenever $\Gamma$ is generated by a maximally monotone mapping $A$ without a full domain. We conclude in this case that $\Gamma$ is maximally $c$-monotone, however, $\Gamma_{1,3}$ is not maximally monotone.

\section{Acknowledgments}

We thank three anonymous referees for their kind and useful remarks. Sedi Bartz was partially supported by a University of Massachusetts Lowell startup grant. Heinz Bauschke and Xianfu Wang were partially supported by the Natural Sciences and Engineering Research Council of Canada. Hung Phan was partially supported by Autodesk, Inc.

\section{References}

[1] M. Agueh and G. Carlier, Barycenters in the Wasserstein Space, SIAM Journal on Mathematical Analysis 43 (2011), 904-924.

[2] S. Bartz and S. Reich, Abstract convex optimal antiderivatives, Annales de l'Institut Henri Poincare (C) Non Linear Analysis 29 (2012), 435-454. 
[3] S. Bartz and S. Reich, Optimal pricing for optimal transport, Set-Valued and Variational Analysis 22 (2014), 467-481.

[4] S. Bartz, H.H. Bauschke and X. Wang, The resolvent order: a unification of the orders by Zarantonello, by Loewner, and by Moreau, SIAM Journal on Optimization 27 (2017), 466-477.

[5] S. Bartz, H.H. Bauschke and X. Wang, A class of multi-marginal c-cyclically monotone sets with explicit c-splitting potentials, Journal of Mathematical Analysis and Applications 461 (2018), 333-348.

[6] H.H. Bauschke and P.L. Combettes, Convex Analysis and Monotone Operator Theory in Hilbert Spaces, 2nd edition, Springer, 2017.

[7] M. Beiglböck and C. Griessler, An optimality principle with applications in optimal transport, arXiv preprint, arXiv:1404.7054 (2014).

[8] H. Brezis, Liquid crystals and energy estimates for $S^{2}$-valued maps, Theory and Applications of Liquid Crystals (Minneapolis, Minn., 1985), The IMA Volumes in Mathematics and its Applications Volume 5, Springer, (1987), 31-52.

[9] G. Carlier, On a class of multidimensional optimal transportation problems, Journal of Convex Analysis 10 (2003), 517-529.

[10] G. Carlier and B. Nazaret, Optimal transportation for the determinant, ESAIM: Control, Optimisation and Calculus of Variations 14 (2008), 678-698.

[11] W. Gangbo and R. McCann, The geometry of optimal transportation, Acta Mathematica 177 (1996), 113-161.

[12] W. Gangbo and A. Swiech, Optimal maps for the multidimensional Monge-Kantorovich problem, Communications on Pure and Applied Mathematics 51 (1998), 23-45.

[13] N. Ghoussoub and B. Maurey, Remarks on multi-marginal symmetric Monge-Kantorovich problems, Discrete and Continuous Dynamical Systems 34 (2013), 1465-1480.

[14] N. Ghoussoub and A. Moameni, Symmetric Monge-Kantorovich problems and polar decompositions of vector fields, Geometric and Functional Analysis 24 (2014), 1129-1166.

[15] C. Griessler, $c$-cyclical monotonicity as a sufficient criterion for optimality in the multimarginal Monge-Kantorovich problem, Proceedings of the American Mathematical Society 146 (2018), 4735-4740.

[16] H.G. Kellerer, Duality theorems for marginal problems, Zeitschrift für Wahrscheinlichkeitstheorie und Verwandte Gebiete 67 (1984), 399-432.

[17] Y.-H. Kim and B. Pass, A general condition for Monge solutions in the multi-marginal optimal transport problem, SIAM Journal on Mathematical Analysis 46 (2014), 1538-1550.

[18] M. Knott and C.S. Smith, On a generalization of cyclic monotonicity and distances among random vectors, Linear Algebra and its Applications 199 (1994), 363-371. 
[19] S. Di Marino, L. De Pascale and M. Colombo, Multimarginal optimal transport maps for 1dimensional repulsive costs, Canadian Journal of Mathematics 67 (2015), 350-368.

[20] S. Di Marino, A. Gerolin and L. Nenna, Optimal transportation theory with repulsive costs, Topological Optimization and Optimal Transport: In the Applied Sciences 9 (2017), 204-256.

[21] B. Pass, On the local structure of optimal measures in the multi-marginal optimal transportation problem, Calculus of Variations and Partial Differential Equations 43 (2012), 529-536.

[22] B. Pass, Multi-marginal optimal transport: theory and applications, ESAIM: Mathematical Modelling and Numerical Analysis 49 (2015), 1771-1790.

[23] J.-C. Rochet, A necessary and sufficient condition for rationalizability in a quasilinear context, Journal of Mathematical Economics 16 (1987), 191-200.

[24] R.T. Rockafellar, Characterization of the subdifferentials of convex functions, Pacific Journal of Mathematics 17 (1966), 497-510.

[25] R.T. Rockafellar and R.J-B. Wets, Variational Analysis, Springer, 1998.

[26] L. Rüschendorf, On c-optimal random variables, Statistics and Probability Letters 27 (1996), 267-270.

[27] L. Rüschendorf and L. Uckelmann, On Optimal multivariate couplings, chapter in Distributions with given Marginals and Moment Problems, Springer (1997), 261-273.

[28] F. Santambrogio, Optimal Transport for Applied Mathematicians, Birkhäuser, 2015.

[29] V. Soloviov, Duality for nonconvex optimization and its applications, Analysis Mathematica 19 (1993), 297-315.

[30] C. Villani, Optimal Transport: Old and New, Springer, 2009. 